\title{
TENSOR PRODUCT OF THE POWER GRAPHS OF SOME FINITE RINGS *
}

\author{
Masoumeh Soleimani, Mohammad Hassan Naderi and Ali Rreza Ashrafi
}

\begin{abstract}
Suppose $R$ is a ring. The multiplicative power graph $\mathcal{P}(R)$ of $R$ is the graph whose vertices are elements of $R$, where two distinct vertices $x$ and $y$ are adjacent if and only if there exists a positive integer $n$ such that $x^{n}=y$ or $y^{n}=x$. In this paper, the tensor product of the power graphs of some finite rings are studied.

Keywords: Power graph; bipartite graph; finite rings; tensor product.
\end{abstract}

\section{Introduction}

All graphs considered here are assumed to be undirected and simple and the vertex and edge set of such a graph $G$ will be denoted by $V(G)$ and $E(G)$, respectively. An edge connecting two vertices $x$ and $y$ in $G$ is denoted by $x y$. We first state some definitions and notations that will be kept throughout the paper.

Given a semigroup $S$, the undirected power graph $\mathcal{P}(S)$ has a vertex set $S$ and two distinct vertices $x$ and $y$ are adjacent if and only if $x^{n}=y$ or $y^{n}=x$, for a positive integer $n$ [4]. The directed version of this graph was introduced by Kelarev and Quinn in an innovating work [11]. These authors continued their work on this graph in papers $[8,9,10]$. We also recommend that the authors should be consulted for the survey article [1] and references therein for more information on this topic. In [14], the authors proved a number of results that relate the structure of the group to the structure of its power graph. Among other things, they presented a counterexample to a conjecture of Charkabarty, Ghosh and Sen. In [3], it was proved that the only finite group whose automorphism group is the same as that of its power graph is the Klein group of order 4.

Mary Flagg [6], in her interesting paper studied the power graph of rings. Since a ring $R$ has two binary operations "+" and " $\times$ ", there will be two different power

Received November 06, 2017; accepted January 14, 2019

2010 Mathematics Subject Classification. Primary 20F12; Secondary 20F14, 20F18, 20D15

* The third author was supported in part by the University of Kashan under grant number $364988 / 222$. 
graphs $\mathcal{P}^{+}(R)$ and $\mathcal{P}^{\times}(R)$ that can be associated to $R$. The power graphs $\mathcal{P}^{+}(R)$ and $\mathcal{P}^{\times}(R)$ are called the additive and multiplicative power graphs of $R$, respectively.

Recall that a graph is said to be connected if for each pair of distinct vertices $x$ and $y$, there is a finite sequence of distinct vertices $x=x_{1}, \cdots, x_{n}=y$ such that each pair $\left(x_{i}, x_{i+1}\right)$ is an edge. A graph without edges is called totally disconnected. For distinct vertices $x$ and $y$, let $\mathrm{d}(x, y)$ be the shortest length of a path connecting $x$ and $y$ and let $d(x, y)=\infty$ if no such path exists. The diameter of $G$ is defined as $\operatorname{diam}(G)=\max \{d(x, y) \mid x, y \in V(G)\}$.

For a graph $G$, the degree of a vertex $x$ in $G$ is the number of edges of $G$ incident with $x$, denoted by $\operatorname{deg}(x)$. A regular graph is a graph that every vertex has the same degree. The graph $G$ is called bipartite with vertex bipartition $\left\{V_{1}, V_{2}\right\}$ if the set of all vertices of $G$ is $V_{1} \cup V_{2}, V_{1} \cap V_{2}=\varnothing$, and each edge of $G$ joins a vertex from $V_{1}$ to a vertex of $V_{2}$. A complete bipartite graph is a bipartite graph containing all edges joining the vertices of $V_{1}$ and $V_{2}$. A complete bipartite graph on vertex sets of sizes $m$ and $n$ is denoted by $K_{m, n}$. If $m=1$ then the resulting graph $K_{1, n}$ is called a star graph.

Suppose $G$ and $H$ are two graphs. We say that $G$ is a subgraph of $H$, when $V(G) \subseteq V(H)$ and $E(G) \subseteq E(H)$. A cycle in $G$ is a subgraph that by deleting one of its edge the resulting subgraph is a path. The girth of $G$, written $\operatorname{gr}(G)$, is the length of the shortest cycle in $G$ and $\operatorname{gr}(G)=\infty$ if $G$ has no cycle. A connected component of an undirected graph is a subgraph in which any two vertices are connected to each other by at least one path and the number of connected components of $G$ is denoted by $\mathcal{C}(G)$.

The tensor product of graphs $G$ and $H$ is denoted by $G \otimes H$, whose vertex set is $V(G) \times V(H)$ and for which vertices $(g, h)$ and $\left(g^{\prime}, h^{\prime}\right)$ are adjacent precisely when $g g^{\prime} \in E(G)$ and $h h^{\prime} \in E(H)$, see [7] for details.

Suppose $p$ is a prime. Fine [5], classified all rings of order $p^{2}$ as follows:

$$
\begin{aligned}
A_{p} & =\left\langle a: p^{2} a=0, a^{2}=a\right\rangle \cong \mathbb{Z}_{p^{2}}, \\
B_{p} & =\left\langle a: p^{2} a=0, a^{2}=p a\right\rangle, \\
C_{p} & =\left\langle a: p^{2}=0, a^{2}=0\right\rangle, \\
D_{p} & =\left\langle a, b: p a=p b=0, a^{2}=a, b^{2}=b, a b=b a=0\right\rangle=\mathbb{Z}_{p} \oplus \mathbb{Z}_{p}, \\
E_{p} & =\left\langle a, b: p a=p b=0, a^{2}=a, b^{2}=b, a b=a, b a=b\right\rangle, \\
F_{p} & =\left\langle a, b: p a=p b=0, a^{2}=a, b^{2}=b, a b=b, b a=a\right\rangle, \\
G_{p} & =\left\langle a, b: p a=p b=0, a^{2}=0, b^{2}=0, a b=b a=a\right\rangle, \\
H_{p} & =\left\langle a, b: p a=p b=0, a^{2}=0, b^{2}=b, a b=b a=0\right\rangle, \\
I_{p} & =\left\langle a, b: p a=p b=0, a^{2}=b, a b=0\right\rangle, \\
J_{p} & =\left\langle a, b: p a=p b=0, a^{2}=b^{2}=0\right\rangle, \\
K_{p} & = \begin{cases}\left\langle a, b: 2 a=2 b=0, a^{2}=a, b^{2}=a+b, a b=b, b a=b\right\rangle & p=2 \\
\left\langle a, b: p a=p b=0, a^{2}=a, b^{2}=j a, a b=b a=b\right\rangle & p \neq 2\end{cases}
\end{aligned}
$$

where $j$ is not a square in $\mathbb{Z}_{p}$. 
Throughout this paper the cardinality of a set $A$ will be denoted by $|A|$ and $K_{n}$ and $U\left(\mathbb{Z}_{p^{2}}\right)$ stand for the complete graph on $n$ vertices and the group of multiplicative units of $\mathbb{Z}_{p^{2}}$, respectively. Our other notations are standard and can be obtained from the books $[2,12,13]$.

\section{The Number of Components}

By [3, Theorem 1], the additive power graph of a ring determines the additive structure of the ring and so we will focus on the multiplicative power graph $\mathcal{P}(R)=\mathcal{P}^{\times}(R)$. In this section we investigate the number of components of the tensor products of two rings $R$ and $S$. Note that the tensor product of graphs are commutative so in this paper we will avoid the repeated cases. If $x \in R, y \in S$, $A \subseteq R$ and $B \subseteq S$ then we define:

$$
\begin{aligned}
& (x, B)=\{(x, b) \mid b \in S\}, \\
& (A, y)=\{(a, y) \mid a \in A\} .
\end{aligned}
$$

Let $p$ be a prime and $R$ be a ring of order $p$. Then as an additive group, $R \cong \mathbb{Z}_{p}$. This implies that there are two rings of order $p$, the ring $\mathbb{Z}_{p}$ and the zero ring on the additive group, denoted by $N_{p}$.

Theorem 2.1. Suppose $p, q$ are primes, $R_{p}$ and $R_{q}$ denote arbitrary rings of order $p$ and $q$, respectively, and $\Gamma=\mathcal{P}\left(R_{p}\right) \otimes \mathcal{P}\left(R_{q}\right)$. Then one of the following statements is hold:

(1) The graph $\Gamma$ has two components, one of them is isomorphic to a complete bipartite graph $K_{(p-1),(q-1)}$ and another one is the star graph $K_{1,(p-1)(q-1)}$.

(2) $\Gamma$ has one or two components and $p+q-1$ isolated vertices.

(3) $\Gamma$ has a bipartite component and $q$ isolated vertices.

(4) $\Gamma$ has two components of the form $K_{1,(q-1)}$ and $q$ isolated vertices.

(5) The graph $\Gamma$ is totally disconnected.

Proof . Since there are two non-isomorphic rings of a prime order, it is enough to consider the graphs $\mathcal{P}\left(\mathbb{Z}_{p}\right) \otimes \mathcal{P}\left(\mathbb{Z}_{q}\right), \mathcal{P}\left(\mathbb{Z}_{p}\right) \otimes \mathcal{P}\left(N_{q}\right)$ and $\mathcal{P}\left(N_{p}\right) \otimes \mathcal{P}\left(N_{q}\right)$. Our main proof will consider three separate cases as follows:

1. If $\Gamma=\mathcal{P}\left(\mathbb{Z}_{p}\right) \otimes \mathcal{P}\left(\mathbb{Z}_{q}\right)$, then for $p=2$ and any prime $q$ the graph $\mathcal{P}\left(\mathbb{Z}_{2}\right) \otimes$ $\mathcal{P}\left(\mathbb{Z}_{q}\right)$ is totally disconnected, since $\mathcal{P}\left(\mathbb{Z}_{2}\right)$ is totally disconnected. If $p, q \neq 2$ except the case that $p=q=3$, then $V\left(\mathcal{P}\left(\mathbb{Z}_{p}\right) \otimes \mathcal{P}\left(\mathbb{Z}_{q}\right)\right)$ has a subset $A=$ $\left\{(0,0),(0, v),(u, 0) \mid u \in V\left(\mathcal{P}\left(\mathbb{Z}_{p}\right)\right), v \in V\left(\mathcal{P}\left(\mathbb{Z}_{q}\right)\right)\right\}$ of size $|A|=p+q-1$ as its isolated vertices. We claim that all other vertices form a component. For every vertex $(x, y) \in V\left(\mathcal{P}\left(\mathbb{Z}_{p}\right) \otimes \mathcal{P}\left(\mathbb{Z}_{q}\right)\right)-A$, we have the following two cases: 
(a) $x, y \neq 1$. Then it is clear that $(x, y)$ and $(1,1)$ are adjacent.

(b) $x=1$ and $y \neq 1$ or vice versa. In this case, a vertex $\left(x^{\prime}, y^{\prime}\right) \in V\left(\mathcal{P}\left(\mathbb{Z}_{p}\right) \otimes\right.$ $\left.\mathcal{P}\left(\mathbb{Z}_{q}\right)\right)-A$ exists such that $(x, y)$ is adjacent with $\left(x^{\prime}, y^{\prime}\right)$ and the last one is adjacent to $(1,1)$. Note that if $p=q=3$, then $\mathcal{P}\left(\mathbb{Z}_{p}\right) \otimes \mathcal{P}\left(\mathbb{Z}_{q}\right)$ has exactly five isolated vertices and two components isomorphic to $K_{2}$.

2. If $\Gamma=\mathcal{P}\left(\mathbb{Z}_{p}\right) \otimes \mathcal{P}\left(N_{q}\right)$, then it is clear that $\mathcal{P}\left(\mathbb{Z}_{2}\right) \otimes \mathcal{P}\left(N_{q}\right)$ is a totally disconnected graph. Let $p \neq 2$. Then $\mathcal{P}\left(\mathbb{Z}_{p}\right) \otimes \mathcal{P}\left(N_{q}\right)$ have $q$ isolated vertices and a bipartite connected component such that one part contains all vertices of the form $\left(V\left(\mathcal{P}\left(\mathbb{Z}_{p}\right)\right)-\{0\}, 0\right)$ and another part contains all vertices of the form $\left(V\left(\mathcal{P}\left(\mathbb{Z}_{p}\right)\right)-\{0\}, V\left(\mathcal{P}\left(N_{q}\right)\right)-\{0\}\right)$. Note that if $p=q=3$ then we obtain three isolated vertices and two star $K_{1, q-1}$ as components.

3. If $\Gamma=\mathcal{P}\left(N_{p}\right) \otimes \mathcal{P}\left(N_{q}\right)$, then in this case the component corresponding to the vertex $(0,0)$ is a star graph $K_{1,(p-1)(q-1)}$, since the vertex 0 is adjacent to all other vertices in $\mathcal{P}\left(N_{p}\right)$. It is now straightforward to verify that the second component is $K_{(p-1),(q-1)}$.

This completes the proof.

There are 11 non-isomorphic rings of order $p^{2}$ and the power graph of these rings have already described by Flagg in [6]. By [6, Corollary 3.1] and [6, Corollary 3.2], $\mathcal{P}\left(A_{p}\right) \cong \mathcal{P}\left(G_{p}\right), \mathcal{P}\left(B_{p}\right) \cong \mathcal{P}\left(I_{p}\right), \mathcal{P}\left(C_{p}\right) \cong \mathcal{P}\left(J_{p}\right)$ and $\mathcal{P}\left(E_{p}\right) \cong \mathcal{P}\left(F_{p}\right)$. Accordingly, it is sufficient to consider the rings $A_{p}, B_{p}, C_{p}, D_{p}, E_{p}, H_{p}$ and $K_{p}$ in order to investigate the tensor product of the power graphs of two rings of order $p^{2}$.

Theorem 2.2. Let $R_{q}$ be a ring of order $q^{2}$. Then

$$
\mathcal{C}\left(\mathcal{P}\left(A_{p}\right) \otimes \mathcal{P}\left(R_{q}\right)\right) \in\left\{2,3,5,6,8,9,11,12,16, p^{2}+2, p^{2}+4\right\} .
$$

Proof . Our main proof will consider seven cases as follows:

1. $R_{q} \cong A_{q}$. We claim that the tensor product graph has five components with the following vertex sets:

$$
\begin{aligned}
& M_{1}=\{(0,0),(\text { npa, mqa }) \mid 1 \leq n \leq p-1,1 \leq m \leq q-1\}, \\
& M_{2}=\{(n p a, 0),(0, m q a) \mid 1 \leq n \leq p-1,1 \leq m \leq q-1\}, \\
& M_{3}=\left\{(n p a, m a) \mid m \in U\left(\mathbb{Z}_{q^{2}}\right), n \in \mathbb{N}\right\}, \\
& M_{4}=\left\{(m a, n q a) \mid m \in U\left(\mathbb{Z}_{p^{2}}\right), n \in \mathbb{N}\right\}, \\
& M_{5}=\left\{\left(m_{1} a, m_{2} a\right) \mid m_{1} \in U\left(\mathbb{Z}_{p^{2}}\right), m_{2} \in U\left(\mathbb{Z}_{q^{2}}\right)\right\} .
\end{aligned}
$$

To prove our claim, we first notice that $M_{i} \cap M_{j}=\varnothing, 1 \leq i \neq j \leq 5$. Since in $\mathcal{P}\left(A_{p}\right)$ we have $(n p a)^{2}=0$ and also all of the vertices of $U\left(\mathbb{Z}_{p^{2}}\right)$ are just connected to some vertices in $U\left(\mathbb{Z}_{p^{2}}\right)$, it is clear that $M_{i}$ for $1 \leq i \leq 5$ composes a component. Note that if $p=q=2$, one can easily check that each of sets $M_{1}$ and $M_{2}$ composes a component and other sets are split into two components. Also if $p=2$ and $q \geq 3$, then $M_{4}$ split into two components and each of the other sets makes a component. 
2. $R_{q} \cong B_{q}$. In this case, we have two components with the following vertex sets:

$$
\begin{aligned}
& M_{1}=\left\{(u, v) \mid u \in U\left(\mathbb{Z}_{p^{2}}\right), \quad v \in V\left(\mathcal{P}\left(B_{q}\right)\right)\right\} \\
& M_{2}=\left\{(u, v) \mid u \in V\left(\mathcal{P}\left(A_{p}\right)\right)-U\left(\mathbb{Z}_{p^{2}}\right), v \in V\left(\mathcal{P}\left(B_{q}\right)\right)\right\}
\end{aligned}
$$

It is clear that $M_{1} \cap M_{2}=\varnothing$. Since vertices in $U\left(\mathbb{Z}_{p^{2}}\right)$ are in a component of $\mathcal{P}\left(A_{p}\right)$, the graph $\mathcal{P}\left(B_{q}\right)$ is a connected graph, every vertex $u \in V\left(\mathcal{P}\left(A_{p}\right)\right)$ $U\left(\mathbb{Z}_{p^{2}}\right)$ has the form $n p a$, where $a$ is a generator of $A_{p}$ and $n \in \mathbb{N}$. Moreover, there is an edge $0(n p a)$ in $\mathcal{P}\left(A_{p}\right)$, where $1 \leq n \leq p-1$. Thus each of $M_{1}$ and $M_{2}$ makes a component.

3. $R_{q} \cong C_{q}$. We claim that $\mathcal{P}\left(A_{p}\right) \otimes \mathcal{P}\left(C_{q}\right)$ has three connected components as follows:

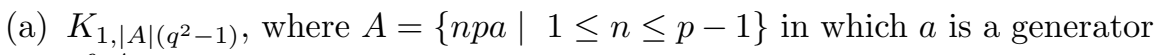
of $A_{p}$.

(b) A complete bipartite graph in which one part is containing all vertices of the form $\left(0, k a^{\prime}\right)$, where $1 \leq k \leq q^{2}-1$ and $a^{\prime}$ is a generator of $C_{q}$. Another part will be the set of all $(n p a, 0), 1 \leq n \leq p-1$.

(c) A bipartite graph in which one part is

$$
\left\{(u, 0) \mid u \in \mathcal{P}\left(A_{p}\right), u \neq n p a, \quad 0 \leq n \leq p-1\right\}
$$

and another part is

$$
\left\{\left(u, k a^{\prime}\right) \mid u \in \mathcal{P}\left(A_{p}\right), u \neq n p a, \quad 0 \leq n \leq p-1,1 \leq k \leq q^{2}-1\right\} .
$$

To prove our claim we note that $(n p a)^{2}=0$ in $\mathcal{P}\left(A_{p}\right)$ and in $\mathcal{P}\left(C_{q}\right)$ the vertex 0 is connected to all other vertices of the form $k a^{\prime}$, for all $1 \leq k \leq$ $q^{2}-1$. Thus, the graph has the edges $\left(n p a, k a^{\prime}\right)(0,0)$ and $\left(0, k a^{\prime}\right)(n p a, 0)$, where $1 \leq n \leq p-1$. In $\mathcal{P}\left(A_{p}\right)$ all vertices that are not multiple of $p$ are in one connected component that completes the assertion. The only exception in this case occurs when $p=q=2$. This special case has the same components and the presented component of (c) is split into two components.

4. $R_{q} \cong D_{q}$. Suppose $\left\{a^{\prime}, b^{\prime}\right\}$ is a generating set for $D_{q}$. It is clear that $\mathcal{P}\left(A_{p}\right) \otimes$ $\mathcal{P}\left(D_{q}\right)$ has $p^{2}$ isolated vertices, since the vertex 0 of $\mathcal{P}\left(D_{q}\right)$ is an isolated vertex. Consider the set of vertices $\left\{\left(k a, i a^{\prime}\right) \mid 0 \leq k \leq p^{2}-1,1 \leq i \leq q-1\right\}$. Then $\left(0, i a^{\prime}\right)$ is adjacent with $\left(n p a, j a^{\prime}\right)$ for all $1 \leq n \leq p-1$ and $1 \leq j \leq q-1$. So, these vertices compose a component except for some values of $p$ and $q$ presenting at the end of this case, the rest vertices of this set make another component corresponding to $(a, a)$. The set $\left\{\left(k a, i a^{\prime}+j b^{\prime}\right) \mid 0 \leq k \leq p^{2}-1,1 \leq\right.$ $i, j \leq q-1\}$ of vertices is partitioned into two sets $\left\{\left(m a, i a^{\prime}+j b^{\prime}\right) \mid m \in\right.$ $\left.U\left(\mathbb{Z}_{p^{2}}\right), \quad 1 \leq i, j \leq q-1\right\}$ and $\left\{\left(n p a, i a^{\prime}+j b^{\prime}\right) \mid 0 \leq n \leq p-1,1 \leq i, j \leq q-1\right\}$ and each of them composes a component. One can easily check that the set 
$\left\{\left(k a, i b^{\prime}\right) \mid 0 \leq k \leq p^{2}-1,1 \leq i \leq q-1\right\}$ also makes a component. Note that there are several exception in this case such that all of them have the same isolated vertices but some differences exist. Now we mention these exceptions. If $p=q=2$ then the tensor product graph is a totally disconnected graph, and also if $p=2$ and $q=3$ then the second set mentioned above is broken up to four star graph $K_{1,3}$, also each of two other sets is partitioned into four components isomorphic to $K_{2}$.

5. $R_{q} \cong E_{q}$. Suppose $\left\{a^{\prime}, b^{\prime}\right\}$ is a generating set for $D_{q}$. In this case, the tensor product graph has eleven components as follows:

(a) Consider the set $\left\{\left(n p a, i a^{\prime}+j b^{\prime}\right) \mid 1 \leq n \leq p-1, \quad 1 \leq i, j \leq q-1, i+j=\right.$ $q$ \}. Since 0 is adjacent with $n p a$ in $\mathcal{P}\left(A_{p}\right), 1 \leq n \leq p-1$, and there are the edges $\left(i a^{\prime}+j b^{\prime}\right) 0$ in $\mathcal{P}\left(E_{q}\right), 1 \leq i, j \leq q-1 ; i+j=q$, this set of vertices only connected to the vertex $(0,0)$. Hence we get a star $K_{1,|A||B|}$, where $A=\{n p a \mid 1 \leq n \leq p-1\}$ is a subset of $V\left(\mathcal{P}\left(A_{p}\right)\right)$ and $B=\left\{i a^{\prime}+j b^{\prime} \mid 1 \leq i, j \leq q-1, i+j=q\right\}$ is a subset of $V\left(\mathcal{P}\left(E_{q}\right)\right)$.

(b) It is easy to check that the vertex set $\left\{\left(n p a, i a^{\prime}\right) \mid 1 \leq i \leq q-1, n \in \mathbb{N}\right\}$ makes a component.

(c) In $E_{q}$ the element $b^{\prime}$ has the same property as $a^{\prime}$, so we obtain a component from $\left\{\left(n p a, i b^{\prime}\right) \mid 1 \leq i \leq q-1, n \in \mathbb{N}\right\}$.

(d) The set $\left\{\left(0, i a^{\prime}+j b^{\prime}\right),(n p a, 0) \mid 1 \leq n \leq p-1,1 \leq i, j \leq q-1, i+j=q\right\}$ forms a component.

(e) The set $\left\{\left(n p a, i a^{\prime}+i b^{\prime}\right) \mid 1 \leq i \leq q-1, n \in \mathbb{N}\right\}$ composes a component. Since in $\mathcal{P}\left(A_{p}\right)$ the vertex 0 is adjacent with $n p a$, where $1 \leq n \leq p-1$ and on the other hand for every vertex $i a^{\prime}+i b^{\prime}$ in $\mathcal{P}\left(E_{q}\right), 1 \leq i \leq q-1$, we have $\left(i a^{\prime}+i b^{\prime}\right)^{2}=2 i^{2} a^{\prime}+2 i^{2} b^{\prime}$. According to the presentation of the ring $E_{q}$, if $1 \leq i \leq q-1$, then $1 \leq 2 i^{2} \leq q-1$ and so we can set $i^{\prime}=2 i^{2}$. Thus in $\mathcal{P}\left(E_{q}\right)$ we have the edges $\left(i a^{\prime}+i b^{\prime}\right)\left(i^{\prime} a^{\prime}+i^{\prime} b^{\prime}\right) ; 1 \leq i, i^{\prime} \leq q-1$.

(f) The set $\left\{(m a, 0),\left(m a, i a^{\prime}+j b^{\prime}\right) \mid m \in U\left(\mathbb{Z}_{p^{2}}\right), 1 \leq i, j \leq q-1, i+j=q\right\}$ makes a bipartite component with parts $V_{1}$ and $V_{2}$ such that $\left|V_{1}\right|=t$ and $\left|V_{2}\right|=t|A|$, where $A=\left\{i a^{\prime}+j b^{\prime} \mid 1 \leq i, j \leq q-1, i+j=q\right\}$ and $t=\left|U\left(\mathbb{Z}_{p^{2}}\right)\right|$.

(g) The set $\left\{\left(m a, k a^{\prime}\right) \mid m \in U\left(\mathbb{Z}_{p^{2}}\right), 1 \leq k \leq q-1\right\}$ is a component.

(h) Similar to the case (g) the set $\left\{\left(m a, k b^{\prime}\right) \mid m \in U\left(\mathbb{Z}_{p^{2}}\right), 1 \leq k \leq q-1\right\}$ makes an isomorphic component.

(i) The set $\left\{\left(m a, i a^{\prime}+i b^{\prime}\right) \mid m \in U\left(\mathbb{Z}_{p^{2}}\right), 1 \leq i \leq q-1\right\}$ is a component, since in $\mathcal{P}\left(A_{p}\right)$ if $m a$ is adjacent to $x$, then $x=m^{\prime} a$, where $m^{\prime} \in U\left(\mathbb{Z}_{p^{2}}\right)$. On the other hand, by using the same argument as in (e), one can show that $\left(i a^{\prime}+i b^{\prime}\right)$ is adjacent to $\left(i^{\prime} a^{\prime}+i^{\prime} b^{\prime}\right)$, where $1 \leq i, i^{\prime} \leq q-1$.

(j) The component corresponding to the set

$$
\left\{\left(n p a, i a^{\prime}+j b^{\prime}\right) \mid 1 \leq i, j \leq q-1, i+j \neq q, i \neq j, n \in \mathbb{N}\right\}
$$


is a bipartite graph with parts $V_{1}$ and $V_{2}$ such that $\left|V_{1}\right|=|A|$ and $\left|V_{2}\right|=|A|(|B|-1)$, where $A=\left\{i a^{\prime}+j b^{\prime} \mid 1 \leq i, j \leq q-1, i+j \neq q\right\}$ and $B=\{n p a \mid n \in \mathbb{N}\}$.

(k) It is obvious that the vertices of the form $\left(m a, i a^{\prime}+j b^{\prime}\right)$ where $m \in$ $U\left(\mathbb{Z}_{p^{2}}\right), 1 \leq i \neq j \leq q-1, i+j \neq p$ make a component.

Note that in this case if $p=q=2$, then the tensor product graph has 8 isolated vertices and four connected components isomorphic to $K_{2}$. If $p=q=3$, then the tensor product graph has exactly nine components.

6. $R_{q} \cong H_{q}$ and $p=q=2$. The graph only contains eight components isomorphic to $K_{2}$. Also if $p=2$ and $q=3$, then the connected component will be introduced in (6.d) splits into two connected components. For other values of $p$ and $q$ it is straightforward to check that one of the following cases will be occurred for the components of $\mathcal{P}\left(A_{p}\right) \otimes \mathcal{P}\left(H_{q}\right)$.

(a) A star graph $K_{1,\left(q^{2}-1\right)}$ containing the vertex $(0,0)$ and vertices $\left(n p a, m a^{\prime}\right)$ for all $1 \leq n \leq p-1$ and $1 \leq m \leq q-1$.

(b) A complete bipartite graph corresponding to the set

$$
\left\{(n p a, 0),\left(0, m a^{\prime}\right) \mid 1 \leq n \leq p-1,1 \leq m \leq q-1\right\}
$$

of vertices.

(c) The set $\left\{(n p, v) \mid 0 \leq n \leq p-1,0 \leq m \leq q-1, v \in V\left(\mathcal{P}\left(H_{q}\right)\right)-\left\{m a^{\prime}\right\}\right\}$ makes a bipartite component.

(d) For every $m \in U\left(\mathbb{Z}_{p^{2}}\right)$, the vertices of the form $(m a, 0)$ is connected to the vertices of the form $\left(m^{\prime} a, k a^{\prime}\right)$, where $m \neq m^{\prime} \in U\left(\mathbb{Z}_{p^{2}}\right), 1 \leq k \leq q-1$. So, these vertices form a component.

(e) The component corresponding to the set

$$
\left\{(u, v) \mid u \in U\left(\mathbb{Z}_{p^{2}}\right), 0 \leq m \leq q-1, v \in V\left(\mathcal{P}\left(H_{q}\right)\right)-\left\{m a^{\prime}\right\}\right\} .
$$

7. $R_{q} \cong K_{q}$. Then the tensor product graph contain $p^{2}$ isolated vertices and two other components.

This completes our argument.

Theorem 2.3. Let $R_{q}$ be a ring of order $q^{2}$. Then

$$
\mathcal{C}\left(\mathcal{P}\left(B_{p}\right) \otimes \mathcal{P}\left(R_{q}\right)\right) \in\left\{1,2,4,5,9,16, p^{2}+1, p^{2}+3\right\} .
$$

Proof . Suppose $a$ is a generator of $B_{p}$. Our main proof will consider six cases as follows: 
1. $R_{q} \cong B_{q}$ and $a^{\prime}$ is a generator of $B_{q}$. In this case, the graph vertices can be partitioned into the parts $M_{i}, 1 \leq i \leq 5$.

$$
\begin{aligned}
& M_{1}=\left\{(u, v) \mid u \in V\left(\mathcal{P}\left(B_{p}\right)\right), v \in V\left(\mathcal{P}\left(B_{q}\right)\right), v \neq t q a^{\prime}, t \in \mathbb{N}\right\}, \\
& M_{2}=\left\{\left(n p a, m q a^{\prime}\right) \mid 0 \leq n \leq p-1,0 \leq m \leq q-1\right\}, \\
& \left.M_{3}=\left\{\left(u, m q a^{\prime}\right) \mid u \in V\left(\mathcal{P}\left(B_{p}\right)\right)-\{n p a\}, 0 \leq n \leq p-1,1 \leq m \leq q-1\right\}\right\} \\
& M_{4}=\left\{\left(a \text { or } k p a, l q a^{\prime}\right) \mid 1 \leq k \leq p-1,1 \leq l \leq q-1\right\}, \\
& M_{5}=\left\{\left(u, l q a^{\prime}\right) \mid u \in V\left(\mathcal{P}\left(B_{p}\right)\right)-\{a, k p a\}, 1 \leq k \leq p-1,1 \leq l \leq q-1\right\},
\end{aligned}
$$

where $n$ and $m$ are squares modulo $p$ and $q$, respectively. Moreover, $k$ and $l$ are not squares modulo $p$ and $q$, respectively. It is easy to check that $M_{i} \cap M_{j}=\varnothing$ and there are vertices $u_{i} \in M_{i}$ and $u_{j} \in M_{j}$ which are adjacent in $B_{p} \times B_{q}$, $1 \leq i, j \leq 5$. Hence this graph is connected.

2. $R_{q} \cong C_{q}$. In this case, $\mathcal{P}\left(B_{p}\right) \otimes \mathcal{P}\left(C_{q}\right)$ is a bipartite graph with parts $V_{1}$ and $V_{2}$ such that $\left|V_{1}\right|=p^{2}\left(q^{2}-1\right)$ and $\left|V_{2}\right|=p^{2}$. Hence this graph is connected.

3. $R_{q} \cong D_{q}$ and $\left\{a^{\prime}, b^{\prime}\right\}$ is a generating set for $D_{q}$. In this case, we claim that the tensor product graph has $p^{2}+3$ components. Since 0 is an isolated vertex of $\mathcal{P}\left(D_{q}\right)$, the tensor product graph has $p^{2}$ isolated vertices. On the other hand, this graph has a bipartite component for $q \neq 2$, such that one part is containing all vertices of the form $\left(u, a^{\prime}+b^{\prime}\right)$ in which $u \in V\left(\mathcal{P}\left(B_{p}\right)\right)$ and another part is the set of all vertices of the form $(u, v)$ such that $u \in V\left(\mathcal{P}\left(B_{p}\right)\right)$ and $v \in V\left(\mathcal{P}\left(D_{q}\right)\right)-A$, where $A=\left\{0, m a^{\prime}, m b^{\prime}, a^{\prime}+b^{\prime} \mid 1 \leq m \leq q-1\right\}$ is a subset of $V\left(\mathcal{P}\left(D_{q}\right)\right)$. Note that $\mathcal{P}\left(B_{p}\right)$ is a connected graph but it is not regular. Also the adjacent vertices to $a^{\prime}+b^{\prime} \in V\left(\mathcal{P}\left(D_{q}\right)\right.$ are the group of unit elements of the ring $D_{q}$. These are all elements of the form $i a^{\prime}+j b^{\prime}$, $1 \leq i, j \leq q-1$, and so each vertex of $V\left(\mathcal{P}\left(B_{p}\right)\right)-A$ is adjacent to $a^{\prime}+b^{\prime}$. Therefore, this component is a non-complete bipartite subgraph. It is clear that the sets $\left\{\left(u, m a^{\prime}\right) \mid u \in V\left(\mathcal{P}\left(B_{p}\right)\right), 1 \leq m \leq q-1\right\}$ and $\left\{\left(u, m b^{\prime}\right) \mid u \in\right.$ $\left.V\left(\mathcal{P}\left(B_{p}\right)\right), 1 \leq m \leq q-1\right\}$ are different components for the graph. Thus, we get exactly $p^{2}+3$ components. One can see that if $p=q=2$ then the tensor product graph is a totally disconnected graph on sixteen vertices.

4. $R_{q} \cong E_{q}$ and $\left\{a^{\prime}, b^{\prime}\right\}$ is a generating set for $E_{q}$. One can see that the graph has five connected components that two of them make from the vertices of the form $\left(u, m a^{\prime}\right)$ and $\left(u, m b^{\prime}\right)$, respectively, where $m \in V\left(\mathcal{P}\left(B_{p}\right)\right), 1 \leq m \leq q-1$. Three other components are corresponding to three set of vertices as follows:

$$
\begin{aligned}
& \left\{\left(u, i a^{\prime}+i b^{\prime}\right) \mid u \in V\left(\mathcal{P}\left(B_{p}\right)\right), 1 \leq i \leq q-1\right\}, \\
& \left\{\left(u, i a^{\prime}+j b^{\prime}\right) \mid u \in V\left(\mathcal{P}\left(B_{p}\right)\right), 1 \leq i \neq j \leq q-1, i+j \neq q\right\}, \\
& \left\{(u, 0),\left(u, i a^{\prime}+j b^{\prime}\right) \mid u \in V\left(\mathcal{P}\left(B_{p}\right)\right), 1 \leq i \neq j \leq q-1, i+j=q\right\} .
\end{aligned}
$$

If $p=q=2$, then the graph has eight isolated vertices and just a component. If $p=2$ and $q=3$ this graph has four components since it does note have the component corresponding to

$$
\left\{\left(u, i a^{\prime}+j b^{\prime}\right) \mid u \in V\left(\mathcal{P}\left(B_{p}\right)\right), 1 \leq i \neq j \leq q-1, i+j \neq q\right\} .
$$


5. $R_{q} \cong H_{q}$. Suppose that $a^{\prime}$ is a generator of $H_{q}$. In this case, the tensor product graph has two components which one of them is containing all vertices in the form $(u, 0)$ and $\left(u, m a^{\prime}\right) ; u \in V\left(\mathcal{P}\left(B_{p}\right)\right)$ and $1 \leq m \leq q-1$. The remaining vertices will make another component.

6. $R_{q} \cong K_{q}$. In this case, all vertices of the form $(u, 0)$ where $u \in V\left(\mathcal{P}\left(B_{p}\right)\right)$ are isolated vertices of the tensor product graph, since 0 is an isolated vertex of $\mathcal{P}\left(K_{q}\right)$. These are $p^{2}$ isolated vertices. On the other hand, all of non-zero vertices are connected to each other in $\mathcal{P}\left(K_{q}\right)$, hence all the remaining vertices put together another component.

Hence the result.

Theorem 2.4. Let $R_{q}$ be a ring of order $q^{2}$. Then

$$
\mathcal{C}\left(\mathcal{P}\left(C_{p}\right) \otimes \mathcal{P}\left(R_{q}\right)\right) \in\left\{2,3,4,6,10, p^{2}+1, p^{2}+3, p^{2}+6,4 p^{2}\right\} .
$$

Proof . Suppose $a$ is a generator of the ring $C_{p}$. Our main proof will consider some cases as follows:

1. $R_{q} \cong C_{q}$. The tensor product graph has a star component isomorphic to $K_{1,3\left(n^{2}-1\right)}$ such that $n=\max \{p, q\}$. Hence the vertex 0 in $\mathcal{P}\left(C_{p}\right)$ is adjacent to every other non-zero vertex. So, $(0,0)$ is adjacent to all vertices in which the first and the second entries are non-zero. Therefore, we obtain a star and a complete bipartite component isomorphic to $K_{p^{2}-1, q^{2}-1}$. Note that all the non-zero vertices of $\mathcal{P}\left(C_{p}\right)$ are connected only with the vertex 0 and so the tensor product has the edges $(u, 0)(0, v)$, where $u, v \in V\left(\mathcal{P}\left(C_{p}\right)\right)-\{0\}$.

2. $R_{q} \cong D_{q}$. Choose a generating set $\left\{a^{\prime}, b^{\prime}\right\}$ for $D_{q}$. The tensor product graph has $p^{2}$ isolated vertices and one can check that the set

$$
\left\{\left(u, m a^{\prime}\right) \mid u \in V\left(\mathcal{P}\left(C_{p}\right)\right), 1 \leq m \leq q-1\right\}
$$

makes a component. Since the elements $a^{\prime}$ and $b^{\prime}$ in $D_{q}$ have the same properties, the set $\left\{\left(u, m b^{\prime}\right) \mid u \in V\left(\mathcal{P}\left(C_{p}\right)\right), 1 \leq m \leq q-1\right\}$ also makes a component isomorphic to last one. So far we do not have considered the vertices of the form $\left(u, i a^{\prime}+j b^{\prime}\right)$, where $u \in V\left(\mathcal{P}\left(C_{p}\right)\right)$ and $1 \leq i, j \leq q-1$. These vertices put together another component. In this case, if $q=2$ then the graph is totally disconnected. If $q=3$ then it is clear that the tensor product has $p^{2}$ isolated vertices and six components.

3. $R_{q} \cong E_{q}$. Suppose $\left\{a^{\prime}, b^{\prime}\right\}$ is a generating set for $D_{q}$. This graph has six components as follows:

(a) A star graph corresponding to the vertex $(0,0)$.

(b) A complete bipartite graph.

(c) The subgraph induced by $\left\{\left(u, m a^{\prime}\right) \mid u \in V\left(\mathcal{P}\left(C_{p}\right)\right), 1 \leq m \leq q-1\right\}$. 
(d) The subgraph induced by $\left\{\left(u, m b^{\prime}\right) \mid u \in V\left(\mathcal{P}\left(C_{p}\right)\right), 1 \leq m \leq q-1\right\}$.

(e) $\left\{(u, 0),\left(0, i a^{\prime}+j b^{\prime}\right) \mid u \in V\left(\mathcal{P}\left(C_{p}\right)\right)-\{0\}, 1 \leq i, j \leq q-1, i+j=q\right\}$.

(f) $\left\{\left(u, i a^{\prime}+j b^{\prime}\right) \mid u \in V\left(\mathcal{P}\left(C_{p}\right)\right), 1 \leq i, j \leq q-1, i \neq j, i+j \neq q\right\}$.

Note that if $p=q=2$ then the graph has ten components.

4. $R_{q} \cong H_{q}$. There is a component corresponding to the vertex $(0,0)$ that is adjacent to all other vertices of the form $\left(n a, m a^{\prime}\right)$, where $1 \leq n \leq p^{2}-1$ and $1 \leq m \leq q-1$. Also the graph has two other components such that each of them can be induced by one of the following subsets:

(a) $\left\{\left(0, m a^{\prime}\right),(n a, 0) \mid 1 \leq m \leq q-1,1 \leq n \leq p^{2}-1\right\}$.

(b) $\left\{(n a, u) \mid u \in V\left(\mathcal{P}\left(H_{q}\right)\right)-\{0, m a\}, 0 \leq n \leq p^{2}-1,1 \leq m \leq q-1\right\}$.

If $q=2$ then the component corresponding to the part (b) will be divided into two new components.

5. $R_{q} \cong K_{q}$. All the vertices of the from $(u, 0)$ where $u \in V\left(\mathcal{P}\left(C_{p}\right)\right)$ are isolated vertices and all of the remaining vertices make only a component.

This completes our argument.

Define:

$T_{1}=\left\{16,18,27,33, p^{2}+q^{2}+8,4 q^{2}, q^{2}+12, q^{2}+7, p^{2}+q^{2}+2,4 p^{2}, q^{2}+9,2 p^{2}+7\right\}$.

Theorem 2.5. Let $R_{q}$ be a ring of order $q^{2}$. Then, $\mathcal{C}\left(\mathcal{P}\left(D_{p}\right) \otimes \mathcal{P}\left(R_{q}\right)\right) \in T_{1}$.

Proof . Suppose $\{a, b\}$ is a generating set for $D_{p}$. Our main proof will consider four cases as follows:

1. $R_{q} \cong D_{q}$. The vertices of the form $(u, 0)$ and $(0, v), u \in V\left(\mathcal{P}\left(D_{p}\right)\right)$ and $v \in V\left(\mathcal{P}\left(D_{q}\right)\right)$, are isolated. It is straightforward to show that each of the set

$$
\begin{aligned}
& \left\{\left(n a, m a^{\prime}\right) \mid 1 \leq n \leq p-1,1 \leq m \leq q-1\right\}, \\
& \left\{\left(n b, m b^{\prime}\right) \mid 1 \leq n \leq p-1,1 \leq m \leq q-1\right\}, \\
& \left\{\left(n a, m b^{\prime}\right) \mid 1 \leq n \leq p-1,1 \leq m \leq q-1\right\}, \\
& \left\{\left(n b, m a^{\prime}\right) \mid 1 \leq n \leq p-1,1 \leq m \leq q-1\right\},
\end{aligned}
$$

induced a component. There are two other components corresponding to the sets

$$
\begin{aligned}
& \left\{\left(a+b, m a^{\prime}\right) \mid 1 \leq m \leq q-1\right\}, \\
& \left\{\left(a+b, m b^{\prime}\right) \mid 1 \leq m \leq q-1\right\},
\end{aligned}
$$

that each of them composes a star isomorphic to $K_{1,\left((p-1)^{2}-1\right) n}$, where $n=$ $\operatorname{deg}\left(m a^{\prime}\right)=\operatorname{deg}\left(m b^{\prime}\right)$. On the other hand, there are two new components 
corresponding to the sets $\left\{\left(n a, a^{\prime}+b^{\prime}\right) \mid 1 \leq n \leq p-1\right\}$ and $\left\{\left(n b, a^{\prime}+b^{\prime}\right) \mid\right.$ $1 \leq n \leq p-1\}$. Obviously, after composing all these connected components all of the remaining vertices are adjacent to the vertex $\left(a+b, a^{\prime}+b^{\prime}\right)$ which gives our final component. In this case, if one of $p$ or $q$ is equal to 2 , then the tensor product is totally disconnected and it has $4 q^{2}$ and $4 p^{2}$ isolated vertices, respectively. Also if $p=q=3$ then the graph has the same components as general case other than the connected component corresponding to the vertex $\left(a+b, a^{\prime}+b^{\prime}\right)$ is broken into two components.

2. $R_{q} \cong E_{q}$. We first notice that the vertices of the form $(0, u), u \in V\left(\mathcal{P}\left(E_{q}\right)\right)$, are isolated vertices in $\mathcal{P}\left(D_{q}\right) \otimes \mathcal{P}\left(E_{q}\right)$. The non-isolated vertices of $\mathcal{P}\left(D_{q}\right) \otimes$ $\mathcal{P}\left(E_{q}\right)$ can be divided into the following sets:

(a) $\left\{\left(n a, m b^{\prime}\right) \mid 1 \leq n \leq p-1,1 \leq m \leq q-1\right\}$,

(b) $\left\{\left(n a, m a^{\prime}\right) \mid 1 \leq n \leq p-1,1 \leq m \leq q-1\right\}$,

(c) $\left\{\left(n b, m a^{\prime}\right) \mid 1 \leq n \leq p-1,1 \leq m \leq q-1\right\}$,

(d) $\left\{\left(n b, m b^{\prime}\right) \mid 1 \leq n \leq p-1,1 \leq m \leq q-1\right\}$,

(e) $\left\{\left(i a+j b, m a^{\prime}\right) \mid 1 \leq i, j \leq p-1,1 \leq m \leq q-1\right\}$,

(f) $\left\{\left(i a+j b, m b^{\prime}\right) \mid 1 \leq i, j \leq p-1,1 \leq m \leq q-1\right\}$,

(g) $\left\{\left(n a, i a^{\prime}+i b^{\prime}\right) \mid 1 \leq n \leq p-1,1 \leq i \leq q-1\right\}$,

(h) $\left\{\left(n b, i a^{\prime}+i b^{\prime}\right) \mid 1 \leq n \leq p-1,1 \leq i \leq q-1\right\}$,

(i) $\{(n a, 0) \mid 1 \leq n \leq p-1\}$,

(j) $\{(n b, 0) \mid 1 \leq n \leq p-1\}$,

(k) $\left\{\left(i a+j b, i^{\prime} a^{\prime}+i^{\prime} b^{\prime}\right) \mid 1 \leq i, j \leq p-1,1 \leq i^{\prime} \leq q-1\right\}$,

(l) $\left\{(i a+j b, 0),\left(i a+j b, i^{\prime} a+j^{\prime} b\right) \mid 1 \leq i, j \leq p-1,1 \leq i^{\prime}, j^{\prime} \leq q-1, i^{\prime}+j^{\prime}=q\right\}$.

One can easily check that each of these subsets induce a component in the graph. The end component is bipartite with vertex bipartization

$$
\begin{aligned}
& \{(i a+j b, 0) \mid 1 \leq i, j \leq p-1\}, \\
& \left\{\left(i a+j b, i^{\prime} a+j^{\prime} b\right) \mid 1 \leq i \neq j \leq p-1,1 \leq i^{\prime}, j^{\prime} \leq q-1, i^{\prime}+j^{\prime}=q\right\} .
\end{aligned}
$$

We now mention some exceptions in this case. If $p=2$ then the graph is totally disconnected with $4 q^{2}$ vertices. If $p \geq 3$ and $q=2$ then the tensor product graph contains $4+2\left(p^{2}-1\right)$ isolated vertices that they are made from the sets $(a-f)$ in above list. Also, each of the next two subsets is broken into two connected components and the remaining vertices composes another connected component. If $p=q=3$ then we have nine isolated vertices and all of the $(a-l)$ are partitioned into two components.

3. $R_{q} \cong H_{q}$. In this case, the tensor product graph has $q^{2}$ isolated vertices $(0, u)$, $u \in V\left(\mathcal{P}\left(H_{q}\right)\right)$. Also, it has seven components corresponding to each of the following subsets:

(a) $\left\{(n a, u) \mid u \in V\left(\mathcal{P}\left(H_{q}\right)\right)-\left\{m a^{\prime}\right\}, 1 \leq n \leq p-1\right\}, 0 \leq m \leq q-1$. 
(b) $\left\{(n b, u) \mid 0 \neq u \in V\left(\mathcal{P}\left(H_{q}\right)\right)-\left\{m a^{\prime}\right\}, 1 \leq n \leq p-1\right\}, 0 \leq m \leq q-1$.

(c) $\left\{\left(a+b, m a^{\prime}\right),(i a+j b, 0) \mid 1 \leq m \leq q-1,1 \leq i, j \leq p-1\right\}$.

(d) $\left\{(a+b, 0),\left(i a+j b, m a^{\prime}\right) \mid 1 \leq m \leq q-1,1 \leq i, j \leq p-1\right\}$.

(e) $\left\{(i a+j b, u) \mid 1 \leq i, j \leq p-1, u \in V\left(\mathcal{P}\left(H_{q}\right)\right)-\left\{m a^{\prime}\right\}, 0 \leq m \leq q-1\right.$.

(f) $\left\{\left(n a, m a^{\prime}\right) \mid 1 \leq n \leq p-1,0 \leq m \leq q-1\right\}$.

(g) $\left\{\left(n b, m a^{\prime}\right) \mid 1 \leq n \leq p-1,0 \leq m \leq q-1\right\}$.

Note that in this case if $p=2$ then the tensor product graph is totally disconnected on $4 q^{2}$ vertices and if $p=q=3$ then all above arguments are valid just the sets (f) and (g) above are divided into two components.

4. $R_{q} \cong K_{q}$. The tenor product graph has $p^{2}+q^{2}+1$ isolated vertices and also each of the set

(a) $\left\{(n a, u) \mid 1 \leq n \leq p-1, u \in V\left(\mathcal{P}\left(H_{q}\right)\right)-\{0\}\right\}$,

(b) $\left\{(n b, u) \mid 1 \leq n \leq p-1, u \in V\left(\mathcal{P}\left(H_{q}\right)\right)-\{0\}\right\}$,

will induce a component. The remaining vertices of this graph compose only one another component, so we have $p^{2}+q^{2}+2$ components. If $p=q=2$, then the tensor product graph is a totally disconnected graph on sixteen vertices.

This proves the theorem.

Theorem 2.6. Let $R_{q}$ be a ring of order $q^{2}$. Then

$$
\mathcal{C}\left(\mathcal{P}\left(E_{p}\right) \otimes \mathcal{P}\left(R_{q}\right)\right) \in\left\{8,10,12,14,20,21, p^{2}+6,2 q^{2}+7\right\} .
$$

Proof . Choose a generating set $\{a, b\}$ for $E_{p}$. The proof will consider four cases as follows:

1. $R_{q} \cong E_{q}$ and $p=q=2$. In this case, we have twelve isolated vertices and two components isomorphic to $K_{2}$. If $p=2$ and $q>2$ then the tensor product graph has $2 q^{2}$ isolated vertices and only seven components. For other values of $p$ and $q$, we don't have isolated vertices and by using the graph structure of $\mathcal{P}\left(E_{p}\right)$, one can check that each of the following subsets induce a unique connected component of the graph:

(a) $\left\{(0,0),\left(i a+j b, i^{\prime} a^{\prime}+j^{\prime} b^{\prime}\right) \mid 1 \leq i, j \leq p-1,1 \leq i^{\prime}, j^{\prime} \leq q-1, i+j=\right.$ $\left.p, i^{\prime}+j^{\prime}=q\right\}$.

(b) $\left\{\left(i a+i b, m a^{\prime}\right) \mid 1 \leq i \leq p-1,1 \leq m \leq q-1\right\}$.

(c) $\left\{\left(0, m a^{\prime}\right),\left(i a+j b, m a^{\prime}\right) \mid 1 \leq i, j \leq p-1, i+j=p, 1 \leq m \leq q-1\right\}$.

(d) $\left.\left(i a+i b, m b^{\prime}\right) \mid 1 \leq i \leq p-1,1 \leq m \leq q-1\right\}$.

(e) $\left\{\left(0, m b^{\prime}\right),\left(i a+j b, m b^{\prime}\right) \mid 1 \leq i, j \leq p-1, i+j=p, 1 \leq m \leq q-1\right\}$. 
(f) $\left\{(i a+j b, 0),\left(0, i^{\prime} a^{\prime}+j^{\prime} b^{\prime}\right) \mid 1 \leq i, j \leq p-1,1 \leq i^{\prime}, j^{\prime} \leq q-1, i+j=\right.$ $\left.p, i^{\prime}+j^{\prime}=q\right\}$, that is a complete bipartite component $K_{p-1, q-1}$.

(g) $\left.\left\{\left(i a+i b, i^{\prime} a^{\prime}+i^{\prime} b^{\prime}\right)\right) \mid, 1 \leq i \leq p-1,1 \leq i^{\prime} \leq q-1\right\}$, that forms a bipartite component, with parts $V_{1}$ and $V_{2}$ such that $\left|V_{1}\right|=\left|V_{2}\right|=t$, where $t=\frac{(p-1)(q-1)}{2}$.

(h) $\left\{\left(i^{\prime} a+j^{\prime} b, i a^{\prime}+j b^{\prime}\right),\left(0, i a^{\prime}+j b^{\prime}\right) \mid 1 \leq i \neq j \leq q-1,1 \leq i^{\prime}, j^{\prime} \leq\right.$ $p-1, i+j \neq q, i^{\prime}+j^{\prime}=p ; \operatorname{deg}\left(i a^{\prime}+j b^{\prime}\right)>\operatorname{deg}\left(j a^{\prime}+i b^{\prime}\right)$ or $\operatorname{deg}\left(i a^{\prime}+j b^{\prime}\right)=$ $\left.\operatorname{deg}\left(j a^{\prime}+i b^{\prime}\right), i<j\right\}$.

(i) $\left\{\left(n a, i a^{\prime}+i b^{\prime}\right) \mid 1 \leq n \leq p-1,1 \leq i \leq q-1\right\}$.

(j) $\left\{\left(n a, i a^{\prime}+j b^{\prime}\right) \mid 1 \leq n \leq p-1,1 \leq i \neq j \leq q-1, i+j \neq q\right\}$.

(k) $\left\{\left(n b, i a^{\prime}+i b^{\prime}\right) \mid 1 \leq n \leq p-1,1 \leq i \leq q-1\right\}$.

(l) $\left\{\left(n b, i a^{\prime}+j b^{\prime}\right) \mid 1 \leq n \leq p-1,1 \leq i \neq j \leq q-1, i+j \neq q\right\}$.

(m) $\left\{\left(n a, m b^{\prime}\right) \mid 1 \leq n \leq p-1,1 \leq m \leq q-1\right\}$.

(n) $\left\{\left(n b, m b^{\prime}\right) \mid 1 \leq n \leq p-1,1 \leq m \leq q-1\right\}$.

(o) $\left\{\left(n a, m a^{\prime}\right) \mid 1 \leq n \leq p-1,1 \leq m \leq q-1\right\}$.

(p) $\left\{\left(n b, m a^{\prime}\right) \mid 1 \leq n \leq p-1,1 \leq m \leq q-1\right\}$.

(q) $\left\{\left(n a, i a^{\prime}+j b^{\prime}\right) \mid 1 \leq n \leq p-1,1 \leq i \neq j \leq q-1\right\}$.

(r) $\left\{\left(i a+i b, i^{\prime} a^{\prime}+j^{\prime} b^{\prime}\right) \mid 1 \leq i \leq p-1,1 \leq i^{\prime} \neq j^{\prime} \leq q-1, i^{\prime}+j^{\prime} \neq q\right\}$.

(s) $\left\{(i a+j b, 0),\left(i a+j b, i^{\prime} a^{\prime}+j^{\prime} b^{\prime}\right) \mid 1 \leq i, j \leq p-1,1 \leq i^{\prime}, j^{\prime} \leq q-1, i+j \neq\right.$ $\left.p, i^{\prime}+j^{\prime}=q\right\}$.

(t) $\left\{(n a, 0),\left(n a, i a^{\prime}+j b^{\prime}\right) \mid 1 \leq n \leq p-1,1 \leq i \neq j \leq q-1, i+j=q\right\}$.

(u) $\left\{(n b, 0),\left(n b, i a^{\prime}+j b^{\prime}\right) \mid 1 \leq n \leq p-1,1 \leq i \neq j \leq q-1, i+j=q\right\}$.

Therefore, we have twenty one connected components.

2. $R_{q} \cong H_{q}$ and $p=q=2$. In this case, the graph has exactly eight isolated vertices, since the vertices $a$ and $b$ are not adjacent in $\mathcal{P}\left(E_{2}\right)$. Also, this graph has four components isomorphic to $K_{2}$. For $p=2$ and $q=3$ it is easy to show that the graph has eighteen isolated vertices and three other components. For other values of $p$ and $q$ we have a star component corresponding to the vertex $(0,0)$. This vertex is adjacent to all vertices of the form $\left(i a+j b, m a^{\prime}\right)$, where $1 \leq i, j \leq p-1, i+j=p$ and $1 \leq m \leq q-1$. Also, it has a bipartite component containing all vertices of the form $(i a+j b, u) \cup(0, u)$, where $1 \leq i, j \leq p-1,1 \leq m \leq q-1$ and $u$ is a non-zero elements of $H_{q}$ such that $u \neq t a^{\prime}, 1 \leq t \leq q-1$. Each of the following sets composes a component:

(a) $\left\{\left(i a+j b, m a^{\prime}\right) \mid 1 \leq i, j \leq p-1, j+j \neq p, 1 \leq m \leq q-1\right\}$

(b) $\left\{\left(0, m a^{\prime}\right),(i a+j b, 0) \mid 1 \leq i, j \leq p-1,1 \leq m \leq q-1, i+j=p\right\}$

(c) $\left\{(n a, u) \mid 1 \leq n \leq p-1,0 \leq m \leq q-1, u \in V\left(\mathcal{P}\left(H_{q}\right)\right)-\left\{m a^{\prime}\right\}\right\}$

(d) $\left\{\left(n a, m a^{\prime}\right) \mid 1 \leq n \leq p-1,0 \leq m \leq q-1\right\}$ 
(e) $\left\{\left(n b, m a^{\prime}\right) \mid 1 \leq n \leq p-1,0 \leq m \leq q-1\right\}$

(f) $\left\{(n b, u) \mid 1 \leq n \leq p-1,0 \leq m \leq q-1, u \in V\left(\mathcal{P}\left(H_{q}\right)\right)-\left\{m a^{\prime}\right\}\right\}$

Therefore, the graph has exactly eight connected components.

3. $R_{q} \cong K_{q}$. In this case, we have $p^{2}$ isolated vertices and six components corresponding to the following subsets:

(a) $\{(0, u),(i a+j b, u) \mid 1 \leq i, j \leq, i+j=p\}$.

(b) $\{(n a, u) \mid 1 \leq n \leq p-1\}$.

(c) $\{(n b, u) \mid 1 \leq n \leq p-1\}$.

(d) $\{(i a+i b, u) \mid 1 \leq i \leq p-1\}$.

(e) $\{(i a+j b, u) \mid 1 \leq i \neq j \leq p-1, i+j \neq p\}$.

(f) $\{(j a+i b, u) \mid(i a+j b, u) \in(e)\}$,

where $u \in V\left(\mathcal{P}\left(K_{q}\right)\right)-\{0\}$. If $p=2$ and $q=2$ or 3 then the graph has exactly 10 or 20 connected components, respectively.

Hence the result.

Theorem 2.7. Let $R_{q}$ be a ring of order $q^{2}$ and $p$ be a prime. Then

$$
\mathcal{C}\left(\mathcal{P}\left(H_{p}\right) \otimes \mathcal{P}\left(R_{q}\right)\right) \in\left\{5,6,8, p^{2}+2\right\}
$$

Proof . Choose the generating set $\{a, b\}$ for $H_{p}$. It is enough to consider two cases that $R_{q} \cong H_{q}$ or $R_{q} \cong K_{q}$.

1. $R_{q} \cong H_{q}$. Consider the following subsets of $H_{p} \otimes R_{q}$ :
(a) $\left\{(0,0),\left(n a, m a^{\prime}\right) \mid 1 \leq n \leq p-1,1 \leq m \leq q-1\right\}$,
(b) $\left\{\left(u, m a^{\prime}\right) \mid 0 \leq m \leq q-1\right\}$,
(c) $\{(u, v)\}$,
(d) $\{(n a, v) \mid 0 \leq n \leq p-1\}$,
(e) $\left\{\left(0, m a^{\prime}\right),(n a, 0) \mid 1 \leq n \leq p-1,1 \leq m \leq q-1\right\}$.

It is easy to see that each of these subset are connected components of $\mathcal{C}\left(\mathcal{P}\left(H_{p}\right) \otimes \mathcal{P}\left(R_{q}\right)\right)$. In each of the cases that $p=q=2$ and $p=2, q=3$ we have the same components that are composed of the set of vertices given in parts $(a)$ and $(e)$ but in the first case each of other set of vertices $(b-d)$ is partitioned into two components isomorphic to $K_{2}$ and in the second case the set of vertices in $(b)$ contains two components.

2. $R_{q} \cong K_{q}$. We can see that $\mathcal{P}\left(H_{p}\right) \otimes \mathcal{P}\left(K_{q}\right)$ has $p^{2}$ isolated vertices and two components corresponding to the subsets: 
(a) $\left\{(n a, u) \mid 0 \leq n \leq p-1, u \in V\left(\mathcal{P}\left(K_{q}\right)\right)-\{0\}\right\}$.

(b) $\left\{(u, v) \mid u \in V\left(\mathcal{P}\left(H_{p}\right)\right)-\{n a\}, 0 \leq n \leq p-1, v \in V\left(\mathcal{P}\left(K_{q}\right)\right)-\{0\}\right\}$.

Therefore, the graph has exactly five, six, eight or $p^{2}+2$ connected components.

This proves the result.

Theorem 2.8. Let $R_{q}$ be a ring of order $q^{2}$. Then $\mathcal{C}\left(\mathcal{P}\left(K_{p}\right) \otimes \mathcal{P}\left(K_{q}\right)\right)=p^{2}+q^{2}$.

Proof . The proof follows from analyzing the graph $\mathcal{P}\left(K_{p}\right)$.

\section{Diameter and Girth}

In Section 2, some information on the connectivity of the tensor product of the power graphs of some ring of order $p^{2}$ were given. In this section our purpose is to obtain diameter of these graphs when they are connected. Furthermore, we will obtain the girth of $\mathcal{P}\left(R_{p}\right) \otimes \mathcal{P}\left(R_{q}\right)$.

Theorem 3.1. Let $R_{p}$ and $R_{q}$ be two rings of order $p^{2}$ and $q^{2}$, respectively. Then the graph $\mathcal{P}\left(R_{p}\right) \otimes \mathcal{P}\left(R_{q}\right)$ is connected if and only if $R_{p} \cong B_{p}$ and $R_{q} \cong B_{q}, C_{q}$. Moreover, $\operatorname{diam}\left(\mathcal{P}\left(B_{p}\right) \otimes \mathcal{P}\left(B_{q}\right)\right)=3$ and $\operatorname{diam}\left(\mathcal{P}\left(B_{p}\right) \otimes \mathcal{P}\left(C_{q}\right)\right)=4$.

Proof . The first part is a direct consequence of Theorems 2.2-2.8. Suppose that $u=\left(x_{1}, y_{1}\right)$ and $v=\left(x_{2}, y_{2}\right)$ are two distinct vertices of the graph $\mathcal{P}\left(B_{p}\right) \otimes \mathcal{P}\left(B_{q}\right)$. With notations as in Theorem 2.3, we have some different cases and in each case we compute $d(u, v)$. In all of the following cases we will introduce the shortest path between $u$ and $v$ in $\mathcal{P}\left(B_{p}\right) \otimes \mathcal{P}\left(B_{q}\right)$.

1. If $u, v \in M_{1}$, then we can assume that there are $1 \leq m_{1}, m_{2}, k_{1}, k_{2} \leq q-1$ such that $y_{1}=\left(m_{1}+k_{1} q\right) a^{\prime}$ and $y_{2}=\left(m_{2}+k_{2} q\right) a^{\prime}$. Since there are not different vertices in the form $(m+k q) a, 1 \leq m, k \leq q-1$, that they are connected in $\mathcal{P}\left(B_{q}\right),\left(x_{1}, y_{1}\right)$ is not adjacent to $\left(x_{2}, y_{2}\right)$. Thus $1<d(u, v)$ and we can proceed based on this fact that whether or not $x_{1}$ is adjacent with $x_{2}$ in $\mathcal{P}\left(B_{p}\right)$. We first assume that $x_{1}$ is adjacent with $x_{2}$ in $\mathcal{P}\left(B_{p}\right)$. Thus $x_{1}=0$ or $x_{2}=0$. Suppose $x_{1}=0$ and choose $1 \leq n, m, k \leq p-1,1 \leq k^{\prime} \leq q-1$. We consider some different cases as follows:

(a) $x_{2}=k p a$, where $k$ is square modulo $p$. We consider the path $u=$ $\left(x_{1}, y_{1}\right)=\left(0,\left(m_{1}+k_{1} q\right) a^{\prime}\right),\left((m+n p) a, k^{\prime} q a^{\prime}\right),\left(k p a,\left(m_{2}+k_{2} q\right) a^{\prime}\right)=$ $\left(x_{2}, y_{2}\right)=v$ of length two, where $k^{\prime}$ is a square modulo $q$.

(b) $x_{2}=k p a$, where $k$ is not square modulo $p$. It is enough to consider the path $u=\left(x_{1}, y_{1}\right)=\left(0,\left(m_{1}+k_{1} q\right) a^{\prime}\right),(t, 0),\left(0, k^{\prime} q a^{\prime}\right),\left(k p a,\left(m_{2}+\right.\right.$ $\left.\left.k_{2} q\right) a^{\prime}\right)=\left(x_{2}, y_{2}\right)=v$ of length three, where $t \in V\left(\mathcal{P}\left(B_{p}\right)\right)-\{0\}$ and $k^{\prime}$ is a square modulo $q$. 
(c) $x_{2}=(m+n p) a$. In this case the path $u=\left(x_{1}, y_{1}\right)=\left(0,\left(m_{1}+k_{1} q\right) a^{\prime}\right)$, $\left(k p a, k^{\prime} q a^{\prime}\right),\left((m+n p) a,\left(m_{2}+k_{2} q\right) a^{\prime}\right)=\left(x_{2}, y_{2}\right)=v$ has length two, where $k$ and $k^{\prime}$ are squares modulo $p$ and $q$, respectively.

Suppose $x_{2}=0$. By a similar method and a case by case investigation, one can see that $d(u, v) \in\{2,3\}$. We now assume that $x_{1}$ is not adjacent to $x_{2}$. It is clear that $x_{1} \neq 0$ and $x_{2} \neq 0$. Choose $1 \leq n, m, k, n^{\prime}, m^{\prime}, k^{\prime} \leq p-1$ and $1 \leq k^{\prime \prime} \leq q-1, k^{\prime \prime}$ is square modulo $q$, based on the following cases:

(a) $x_{1}=(m+n p) a$ and $x_{2}=k p a, k$ is not square modulo $p$. The path $u=\left(x_{1}, y_{1}\right)=\left((m+n p) a,\left(m_{1}+k_{1} q\right) a^{\prime}\right),\left(0, k^{\prime \prime} q a^{\prime}\right),\left(k p a,\left(m_{2}+k_{2} q\right) a^{\prime}\right)=$ $\left(x_{2}, y_{2}\right)=v$ has length two.

(b) $x_{1}=k p a, k$ is not square modulo $p$ and $x_{2}=(m+n p) a$. The path $u=\left(x_{1}, y_{1}\right)=\left(k p a,\left(m_{1}+k_{1} q\right) a^{\prime}\right),\left(0, k^{\prime \prime} q a^{\prime}\right),\left((m+n p) a,\left(m_{2}+k_{2} q\right) a^{\prime}\right)=$ $\left(x_{2}, y_{2}\right)=v$ has length two.

(c) $x_{1}=k p a, k$ is square modulo $p$ and $x_{2}=k^{\prime} p a, k^{\prime}$ is not square modulo $p$. The path $u=\left(x_{1}, y_{1}\right)=\left(k p a,\left(m_{1}+k_{1} q\right) a^{\prime}\right),\left(0, k^{\prime \prime} q a^{\prime}\right),\left(k^{\prime} p a,\left(m_{2}+\right.\right.$ $\left.\left.k_{2} q\right) a^{\prime}\right)=\left(x_{2}, y_{2}\right)=v$ has length two.

(d) $x_{1}=k p a, k$ is not square modulo $p$, and $x_{2}=k^{\prime} p a, k^{\prime}$ is a square modulo $p$. It can be easily seen that the path $u=\left(x_{1}, y_{1}\right)=\left(k p a,\left(m_{1}+k_{1} q\right) a^{\prime}\right)$, $\left(0, k^{\prime \prime} q a^{\prime}\right),\left(k^{\prime} p a,\left(m_{2}+k_{2} q\right) a^{\prime}\right)=\left(x_{2}, y_{2}\right)=v$ has length two.

(e) $x_{1}=(m+n p) a$ and $x_{2}=\left(m^{\prime}+n^{\prime} p\right) a$. It is easy to see that the path $u=\left(x_{1}, y_{1}\right)=\left((m+n p) a,\left(m_{1}+k_{1} q\right) a^{\prime}\right),\left(k^{\prime} p a, k^{\prime \prime} q a^{\prime}\right),\left(\left(m^{\prime}+n^{\prime} p\right) a,\left(m_{2}+\right.\right.$ $\left.\left.k_{2} q\right) a^{\prime}\right)=\left(x_{2}, y_{2}\right)=v$ has length three, where $k^{\prime}$ is a square modulo $p$.

(f) $x_{1}=k p a$, and $x_{2}=k^{\prime} p a, k$ and $k^{\prime}$ are squares modulo $p$ and $q$, respectively. The path $u=\left(x_{1}, y_{1}\right)=\left(k p a,\left(m_{1}+k_{1} q\right) a^{\prime}\right),(0$ or $(m+$ $\left.n p) a, k^{\prime \prime} q a^{\prime}\right),\left(k^{\prime} p a,\left(m_{2}+k_{2} q\right) a^{\prime}\right)=\left(x_{2}, y_{2}\right)=v$ has length two.

(g) $x_{1}=k p a$, and $x_{2}=k^{\prime} p a, k$ and $k^{\prime}$ are not squares modulo $p$ and $q$, respectively. The path $u=\left(x_{1}, y_{1}\right)=\left(k p a,\left(m_{1}+k_{1} q\right) a^{\prime}\right),\left(0, k^{\prime \prime} q a^{\prime}\right)$, $\left(k^{\prime} p a,\left(m_{2}+k_{2} q\right) a^{\prime}\right)=\left(x_{2}, y_{2}\right)=v$ has length two.

2. $u, v \in M_{2}$. A similar argument as in the Case 1 shows that $d(u, v) \in\{1,2\}$.

3. $u, v \in M_{4}$. Since $d(u, v)>1$, the only case that can be occurred is the case that in $\mathcal{P}\left(B_{p}\right), x_{1}$ is not adjacent to $x_{2}$ and in $\mathcal{P}\left(B_{q}\right), x_{2}$ is not adjacent to $y_{2}$. Choose the path $\left(x_{1}, y_{1}\right),(0,0),\left(x_{2}, y_{2}\right)$ of length two to prove that $d(u, v)=2$.

Note that a similar argument for the remaining cases shows that $d(u, v) \in\{1,2,3\}$ and so $\operatorname{diam}\left(\mathcal{P}\left(B_{p}\right) \otimes \mathcal{P}\left(B_{q}\right)\right)=3$. We now return to determine $\operatorname{diam}\left(\mathcal{P}\left(B_{p}\right) \otimes\right.$ $\mathcal{P}\left(C_{q}\right)$ ). By Theorem 2.3, the graph $\mathcal{P}\left(B_{p}\right) \otimes \mathcal{P}\left(C_{q}\right)$ is bipartite and the parts $V_{1}$ and $V_{2}$ are defined as the set of all vertices of the form $\left(V\left(\mathcal{P}\left(B_{p}\right)\right), 0\right)$ and $\left(V\left(\mathcal{P}\left(B_{p}\right)\right), V\left(\mathcal{P}\left(C_{q}\right)\right)-\{0\}\right)$, respectively. It is obvious that if $u$ and $v$ are in different parts, then $d(u, v) \in\{1,3\}$. If $u, v \in V_{1}$, then one can see that $d(u, v) \in$ $\{2,4\}$. Assume that $u, v \in V_{2}$, where $u=\left(u_{1}, v_{1}\right)$ and $v=\left(u_{2}, v_{2}\right)$ such that $u_{1}$ and $u_{2}$ are non-zero. Then the path $\left(u_{1}, v_{1}\right),(0,0),\left(u_{2}, v_{2}\right)$, connecting $u$ and $v$ 
is a shortest path in this case. If $u_{1}$ and $u_{2}$ are zero, then we consider the path $\left(u_{1}, v_{1}\right),(k p a, 0),\left(u_{2}, v_{2}\right)$, where $1 \leq k \leq p-1$ and $k$ is a square modulo $p$ and so $d(x, y)=2$. Therefore, $\operatorname{diam}\left(\mathcal{P}\left(B_{p}\right) \otimes \mathcal{P}\left(C_{q}\right)\right)=4$.

Theorem 3.2. Let $R_{q}$ be a ring of order $q^{2}$. Then $\operatorname{gr}\left(\mathcal{P}\left(A_{p}\right) \otimes \mathcal{P}\left(R_{q}\right)\right) \in\{3,4,6, \infty\}$.

Proof . Apply Theorems 2.3-2.8. We have the following separate cases:

1. $R_{q} \cong A_{q}$ and $p, q \neq 2$. It is clear that we have the cycle $(u, v),(x, y)$, $\left(x^{-1}, y^{-1}\right),(u, v)$, where $u \in U\left(\mathbb{Z}_{p^{2}}\right), v \in U\left(\mathbb{Z}_{q^{2}}\right), x$ is a generator of $U\left(\mathbb{Z}_{p^{2}}\right)$ and $y$ is a generator of $U\left(\mathbb{Z}_{q^{2}}\right)$. Thus, the girth of the graph is 3 .

2. $R_{q} \cong B_{q}$ and $p, q \neq 2$. In this case let $u \in U\left(\mathbb{Z}_{p^{2}}\right), v$ be a generator of $U\left(\mathbb{Z}_{p^{2}}\right)$ and $1 \leq m, k \leq q-1$. Then we will have the following cycles:

(a) $\left(u, k q a^{\prime}\right),(v, 0),\left(v^{-1},(m+k q) a^{\prime}\right),\left(u, k q a^{\prime}\right)$,

(b) $(u, 0),\left(v, k q a^{\prime}\right),\left(v^{-1},(m+k q) a^{\prime}\right),(u, 0)$,

(c) $\left(u,(m+k q) a^{\prime}\right),\left(v, k q a^{\prime}\right),\left(v^{-1}, 0\right),\left(u,(m+k q) a^{\prime}\right)$.

Hence the girth of the graph will be 3 .

3. $R_{q} \cong D_{q}, p \neq 2$ and $p, q \neq 3$. In this case let $u \in U\left(\mathbb{Z}_{p^{2}}\right), v$ be a generator of $U\left(\mathbb{Z}_{p^{2}}\right)$ and $1 \leq n, m, k \leq q-1$. Then the shortest cycles have one of the following forms:

(a) $\left(u, n a^{\prime}\right),\left(v, m a^{\prime}\right),\left(v^{-1}, k a^{\prime}\right),\left(u, n a^{\prime}\right)$,

(b) $\left(u, n b^{\prime}\right),\left(v, m b^{\prime}\right),\left(v^{-1}, k b^{\prime}\right),\left(u, n b^{\prime}\right)$.

So, the girth of the graph is 3 .

4. $R_{q} \cong E_{q}, p \neq 2$ and $q \neq 2,3$. Let $u \in U\left(\mathbb{Z}_{p^{2}}\right), v$ be a generator of $U\left(\mathbb{Z}_{p^{2}}\right)$ and $1 \leq n, m, k \leq q-1$. Then the shortest cycles have one of the following forms:

(a) $\left(u, n a^{\prime}\right),\left(v, m a^{\prime}\right),\left(v^{-1}, k a^{\prime}\right),\left(u, n a^{\prime}\right)$,

(b) $\left(u, n b^{\prime}\right),\left(v, m b^{\prime}\right),\left(v^{-1}, k b^{\prime}\right),\left(u, n b^{\prime}\right)$,

and so the girth is equal to 3 .

5. $\quad R_{q} \cong H_{q}$ and $p, q \neq 2$. Let $u \in U\left(\mathbb{Z}_{p^{2}}\right), v$ be a generator of $U\left(\mathbb{Z}_{p^{2}}\right)$. Then the cycle $\left(u, 2 b^{\prime}\right),\left(v, b^{\prime}\right),\left(v^{-1}, 2 a^{\prime}+2 b^{\prime}\right),\left(u, 2 b^{\prime}\right)$ has the shortest length and so the girth is 3 .

6. $R_{q} \cong K_{q}$ and $p \neq 2$. One can see that the cycle $\left(u, a^{\prime}+b^{\prime}\right),\left(v, a^{\prime}\right),\left(v^{-1}, b^{\prime}\right),\left(u, a^{\prime}+\right.$ $\left.b^{\prime}\right)$ has the minimum length. Thus the girth is 4 .

We now present the cases that $\operatorname{gr}\left(\mathcal{P}\left(A_{p}\right) \otimes \mathcal{P}\left(R_{q}\right)\right)=4$. 
$1^{\prime} R_{q} \cong A_{q}, p=2$ and $q \neq 2$. The cycle $(0, u),(2 a, v),\left(0, a^{\prime}\right),\left(2 a, v^{-1}\right),(0, u)$ has the shortest length, where $u \in U\left(\mathbb{Z}_{q^{2}}\right)$ and $v$ is a generator of $\left(U\left(\mathbb{Z}_{q^{2}}\right), \times\right)$.

$2^{\prime} R_{q} \cong A_{q}, q=2$ and $p \neq 2$. Note that $(u, 0),\left(v, 2 a^{\prime}\right),(a, 0),\left(v^{-1}, 2 a^{\prime}\right),(u, 0)$ is a shortest cycle for the graph.

$3^{\prime} R_{q} \cong C_{q}, p \neq 2$. It is enough to consider the following cycles:

(a) $(u, 0),(v, w),\left(v^{-1}, 0\right),(v, z),(u, 0)$,

(b) $(k p a, 0),(0, w),\left(k^{\prime} p a, 0\right),(0, z),(k p a, 0)$,

(c) $(u, w),(v, 0),(a, z),\left(v^{-1}, 0\right),(u, w)$,

where $u \in U\left(\mathbb{Z}_{p^{2}}\right), v$ is a generator of $\left(U\left(\mathbb{Z}_{p^{2}}\right), \times\right), w, z \in V\left(\mathcal{P}\left(C_{q}\right)\right)-\{0\}$, $w \neq z$ and $1 \leq k \neq k^{\prime} \leq p-1$.

$4^{\prime} R_{q} \cong D_{q}, p \neq 2$ and $q=3$. It is enough to choose the shortest cycle $\left(u, a^{\prime}\right)$, $\left(v, 2 a^{\prime}\right),\left(a, a^{\prime}\right),\left(v^{-1}, 2 a^{\prime}\right),\left(u, a^{\prime}\right)$, where $u \in U\left(\mathbb{Z}_{p^{2}}\right)-\{a\}$ and $v$ is a generator of $\left(U\left(\mathbb{Z}_{p^{2}}\right), \times\right)$.

$5^{\prime} R_{q} \cong E_{q}, p \neq 2$ and $q=3$. The cycles:

(a) $\left(u, a^{\prime}\right),\left(v, 2 a^{\prime}\right),\left(a, a^{\prime}\right),\left(v^{-1}, 2 a^{\prime}\right),\left(u, a^{\prime}\right)$,

(b) $\left(u, b^{\prime}\right),\left(v, 2 b^{\prime}\right),\left(a, b^{\prime}\right),\left(v^{-1}, 2 b^{\prime}\right),\left(u, b^{\prime}\right)$,

where $u \in U\left(\mathbb{Z}_{p^{2}}\right)-\{a\}, v$ is a generator of $U\left(\mathbb{Z}_{p^{2}}\right)$, have length 4 and they are the shortest cycles.

$6^{\prime} R_{q} \cong E_{q}, p \neq 2$ and $q=2$. A shortest cycle for the graph is $(u, 0),\left(v, a^{\prime}+b^{\prime}\right)$, $(a, 0),\left(v^{-1}, a^{\prime}+b^{\prime}\right),(u, 0)$, as desired.

$7^{\prime} R_{q} \cong H_{q}, p \neq 2$ and $q=2$. The cycle $(u, 0),\left(v, a^{\prime}\right),(a, 0),\left(v^{-1}, a^{\prime}\right),(u, 0)$ has the shortest length.

$8^{\prime} R_{q} \cong B_{q}$ and $p=2$. The result follows from the fact that $\left(a, q a^{\prime}\right),\left(3 a, a^{\prime}\right)$, $(a, 0),\left(3 a,(q+1) a^{\prime}\right),\left(a, q a^{\prime}\right)$ is a shortest cycle of length 4 .

$9^{\prime} \quad R_{q} \cong C_{q}$ and $p, q \neq 2$. By Theorem 2.2 , the graph $\mathcal{P}\left(A_{p}\right) \otimes \mathcal{P}\left(C_{q}\right)$ has at least one complete bipartite component and so the girth of this graph is 4 .

$10^{\prime} R_{q} \cong H_{q}$ and $p=2$. In this case, a shortest cycle for the graph is $\left(a, b^{\prime}\right)$, $\left(3 a, a^{\prime}+2 b^{\prime}\right),\left(a, 2 b^{\prime}\right),\left(3 a, 2 a^{\prime}+2 b^{\prime}\right),\left(a, b^{\prime}\right)$.

Finally if $R_{q} \cong K_{q}$ and $p=q=2$, then the cycles:

1. $\left(0, a^{\prime}\right),\left(2 a, a^{\prime}+b^{\prime}\right),\left(0, b^{\prime}\right),\left(2 a, a^{\prime}\right),\left(0, a^{\prime}+b^{\prime}\right),\left(2 a, b^{\prime}\right),\left(0, a^{\prime}\right)$

2. $\left(a, a^{\prime}\right),\left(3 a, a^{\prime}+b^{\prime}\right),\left(a, b^{\prime}\right),\left(3 a, a^{\prime}\right),\left(a, a^{\prime}+b^{\prime}\right),\left(3 a, b^{\prime}\right),\left(a, a^{\prime}\right)$,

are the shortest cycles of length 6 . In the remaining cases, the graph is acyclic which completes the proof. 
Theorem 3.3. Let $R_{q}$ be a ring of order $q^{2}$. Then $\operatorname{gr}\left(\mathcal{P}\left(B_{q}\right) \otimes \mathcal{P}\left(R_{q}\right)\right) \in\{3,4, \infty\}$.

Proof . The proof runs as Theorem 3.2. We first note that $(0,0),(a, a),(p a, q a)$, $(0,0)$ is a triangle in $\mathcal{P}\left(B_{p}\right) \otimes \mathcal{P}\left(B_{q}\right)$ and so $\operatorname{gr}\left(\mathcal{P}\left(B_{p}\right) \otimes \mathcal{P}\left(B_{q}\right)\right)=3$. Also, by [2, Theorem 1] we have $\operatorname{gr}\left(\mathcal{P}\left(B_{p}\right) \otimes \mathcal{P}\left(C_{q}\right)\right) \in\{4,6,8\}$, but we have a square $(0,0)$, $(a, a),(p a, 0),(a, q a),(0,0)$ in the graph. Thus $\operatorname{gr}\left(\mathcal{P}\left(B_{p}\right) \otimes \mathcal{P}\left(C_{q}\right)\right)=4$. By Theorem 2.3 , it is straightforward to see that $\operatorname{gr}\left(\mathcal{P}\left(B_{2}\right) \otimes \mathcal{P}\left(D_{2}\right)\right)=\infty$ and for other values of $p$ and $q, \operatorname{gr}\left(\mathcal{P}\left(B_{p}\right) \otimes \mathcal{P}\left(D_{q}\right)\right)=4$, since $\left(0, a^{\prime}\right),\left(a,(q-1) a^{\prime}\right),\left(p a, a^{\prime}\right),((p+1) a,(q-$ 1) $\left.a^{\prime}\right),\left(0, a^{\prime}\right)$ is a shortest cycle for the graph. Furthermore, $\operatorname{gr}\left(\mathcal{P}\left(B_{2}\right) \otimes \mathcal{P}\left(E_{2}\right)\right)=3$ and by Theorem 2.3, for other values of $p$ and $q$ we have the cycle $\left(k p a, i a^{\prime}+i b^{\prime}\right)$, $\left((m+n p) a, a^{\prime}+b^{\prime}\right),\left(0, i^{\prime} a^{\prime}+i^{\prime} b^{\prime}\right),\left(k^{\prime} p a, i a^{\prime}+j b^{\prime}\right)$, where $1 \leq m, n, k, k^{\prime} \leq p-1$, $2 \leq i, i^{\prime} \leq q-1$ and $k$ is a square modulo $p$. In $\mathcal{P}\left(B_{2}\right) \otimes \mathcal{P}\left(H_{2}\right)$, we have the cycle $\left(a, a^{\prime}\right),(2 a, 0),\left(3 a, a^{\prime}\right),(0,0),\left(a, a^{\prime}\right)$ and so $\operatorname{gr}\left(\mathcal{P}\left(B_{2}\right) \otimes \mathcal{P}\left(H_{2}\right)\right)=4$. For other values of $p$ and $q$, we have $\operatorname{gr}\left(\mathcal{P}\left(B_{p}\right) \otimes \mathcal{P}\left(H_{q}\right)\right)=3$, since $\left(a, b^{\prime}\right),\left(p a, 2 b^{\prime}\right),\left(0,2 a^{\prime}+2 b^{\prime}\right),\left(a, b^{\prime}\right)$ is a cycle in the graph. Finally, let $1 \leq m, k, k^{\prime} \leq p-1$ such that $k^{\prime}$ be a square modulo $p$. Then the cycle $\left(0, b^{\prime}\right),\left(k^{\prime} p a, a^{\prime}\right),\left((m+k p) a, a^{\prime}+b^{\prime}\right),\left(0, b^{\prime}\right)$ is a triangle in $\mathcal{P}\left(B_{p}\right) \otimes \mathcal{P}\left(K_{q}\right)$, which proves that $\operatorname{gr}\left(\mathcal{P}\left(B_{p}\right) \otimes \mathcal{P}\left(K_{q}\right)\right)=3$.

Theorem 3.4. Let $R_{q}$ be a ring of order $q^{2}$. Then $\operatorname{gr}\left(\mathcal{P}\left(C_{p}\right) \otimes \mathcal{P}\left(R_{q}\right)\right) \in\{4, \infty\}$.

Proof . By Theorem 2.4, it is easy to prove that if $q=2$, then $\operatorname{gr}\left(\mathcal{P}\left(C_{p}\right) \otimes\right.$ $\left.\mathcal{P}\left(D_{2}\right)\right)=\operatorname{gr}\left(\mathcal{P}\left(C_{p}\right) \otimes \mathcal{P}\left(H_{2}\right)\right)=\infty$ and if $p=q=2$, then $\operatorname{gr}\left(\mathcal{P}\left(C_{p}\right) \otimes \mathcal{P}\left(E_{q}\right)\right)=\infty$. Again by Theorem 2.4 and using the method of Theorem 3.3, we can show that in the remaining cases $\operatorname{gr}\left(\mathcal{P}\left(C_{p}\right) \otimes \mathcal{P}\left(R_{q}\right)\right)=4$.

Theorem 3.5. Let $R_{q}$ be a ring of order $q^{2}$. Then $\operatorname{gr}\left(\mathcal{P}\left(S_{p}\right) \otimes \mathcal{P}\left(R_{q}\right)\right) \in\{4,6, \infty\}$, where $S_{p} \cong D_{p}, E_{p}$ or $H_{p}$ and $R_{q} \cong D_{q}, E_{q}, H_{q}$ or $K_{q}$. Moreover $\operatorname{gr}\left(\mathcal{P}\left(K_{p}\right) \otimes\right.$ $\left.\mathcal{P}\left(K_{q}\right)\right)=3$.

Proof . In view of Theorems 2.5, 2.6 and 2.7, it is clear that if $\left(S_{p} \cong D_{p}, R_{q} \cong\right.$ $D_{q}, K_{q}$ and $p=2$ or $\left.q=2\right),\left(S_{p} \cong E_{p}, R_{q} \cong E_{q}, H_{q}\right.$ and $\left.p=q=2\right)$ and finally $\left(S_{p} \cong H_{p}, R_{q} \cong H_{q}\right.$ and $\left.p=q=2\right)$, then $\operatorname{gr}\left(\mathcal{P}\left(S_{p}\right) \otimes \mathcal{P}\left(R_{q}\right)\right)=\infty$ and also $\operatorname{gr}\left(\mathcal{P}\left(D_{2}\right) \otimes \mathcal{P}\left(H_{q}\right)\right)=\infty$. Also, there is a cycle of length 6 in $\mathcal{P}\left(E_{2}\right) \otimes \mathcal{P}\left(K_{2}\right)$. Moreover, $\mathcal{P}\left(H_{p}\right) \otimes \mathcal{P}\left(H_{q}\right)$, when $p \neq 2$ and $q \neq 2$ has the girth 4 . By the same way in other cases, we have a cycle of length 4 or 6 . To prove the second part, it is enough to consider the triangle $\left(a+b, a^{\prime}+b^{\prime}\right),\left(b, a^{\prime}\right),\left(a, b^{\prime}\right),\left(a+b, a^{\prime}+b^{\prime}\right)$.

\section{Concluding Remarks}

In this paper the number of connected components in the tensor product of the power graphs of some finite rings were computed. We apply our results to calculate the diameter of all such graphs when they are connected. Moreover, the girth of these graphs are also computed. 
In the end of this paper, we suppose that $p, q$ are primes and $R_{p}, R_{q}$ denote arbitrary rings of order $p^{2}$ and $q^{2}$, respectively. Then we claim that $\mathcal{P}\left(R_{p} \times R_{q}\right) \subseteq$ $\mathcal{P}\left(R_{p}\right) \otimes \mathcal{P}\left(R_{q}\right)$. To do this, we first note that for every edge $(a, b)(c, d) \in E\left(\mathcal{P}\left(R_{p} \times\right.\right.$ $\left.R_{q}\right)$ ), there exists $n \in \mathbb{N}$ such that $(a, b)^{n}=(c, d)$ or there exists $m \in \mathbb{N}$ such that $(c, d)^{m}=(a, b)$. Therefore, $a^{n}=c, b^{n}=d$ or $c^{m}=a, d^{m}=b$. Then $a c \in E\left(\mathcal{P}\left(R_{p}\right)\right), b d \in E\left(\mathcal{P}\left(R_{q}\right)\right)$, which shows that $(a, b)(c, d) \in \mathcal{P}\left(R_{p}\right) \otimes \mathcal{P}\left(R_{q}\right)$. The Figures 1 and 2 , present a counterexample which proves that another conclusion does not hold in general.
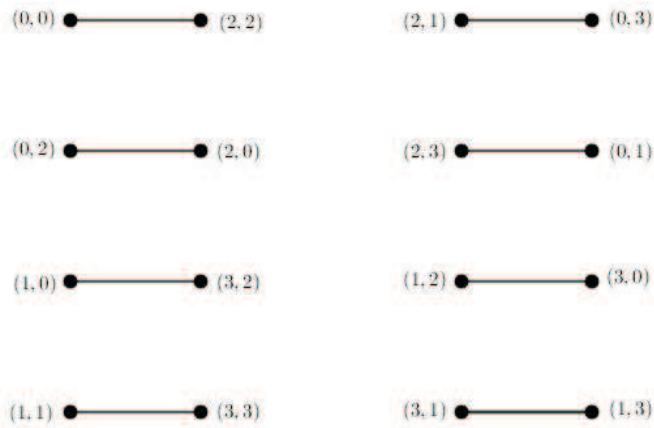

Figure 1. $\mathcal{P}\left(A_{2}\right) \otimes \mathcal{P}\left(A_{2}\right)$
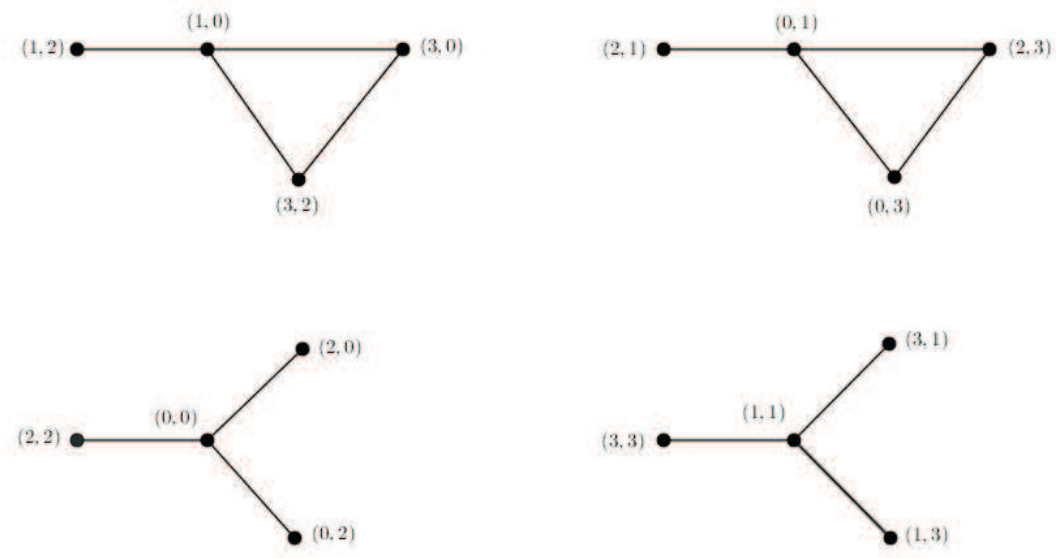

Figure 2. $\mathcal{P}\left(A_{2} \times A_{2}\right)$

Acknowledgement. The authors are indebted to Dr. Mary Flagg from the University of St. Thomas, USA for providing us a pdf of her unpublished paper [6]. 


\title{
R E F E R E N C E S
}

1. J. Abawajy, A. V. Kelarev and M. Chowdhury: Power graphs: a survey. Elec. J. Graph Theory Appl. 1 (2013) (2), 125-147.

2. B. Bollabas: Graph Theory, An Introductory Course. Springer, New York, 1979.

3. P. J. CAmeron: The power graph of a finite group, II. J. Group Theory 13 (2010), 779-783.

4. I. Chakrabarty, S. Ghosh and M. K. Sen: Undirected power graphs of semigroups. Semigroup Forum 78 (3) (2009), 410-426.

5. B. Fine: Classification of finite rings of order $p^{2}$. Math. Mag. 66 (1993), 249252.

6. M. FlaGG: Power graphs of rings. Preprint 2014.

7. R. Hammack, W. Imrich and S. KlavžAR: Handbook of Product Graphs. CRC Press, Taylor and Francis Group, Second Edition, 2011.

8. A. V. Kelarev and S. J. Quinn: A combinatorial property and power graphs of semigroups. Comment. Math. Univ. Carolin. 45 (1) (2004), 1-7.

9. A. V. Kelarev and S. J. Quinn: Directed graphs and combinatorial properties of semigroups. J. Algebra 251 (1) (2002), 16-26.

10. A. V. Kelarev, S. J. Quinn and R. Smolíková: Power graphs and semigroups of matrices. Bull. Austral. Math. Soc. 63 (2) (2001), 341-344.

11. A. V. Kelarev and S. J. Quinn: A combinatorial property and power graphs of groups. Contributions to General Algebra 12 (Vienna, 1999), Heyn, Klagenfurt, 2000, pp. 229-235.

12. A. V. Kelarev: Graph Algebras and Automata. Marcel Dekker, New York, 2003.

13. A. V. Kelarev: Ring Constructions and Applications. World Scientific, River Edge, NJ, 2002.

14. G. R. Pourgholi, H. Yousefi-Azari and A. R. Ashrafi: The undirected power graph of a finite group. Bull. Malays. Math. Sci. Soc. 38 (4) (2015), 15171525.

\author{
Masoumeh Soleimani \\ Faculty of Science \\ Department of Mathematics \\ University of Qom \\ Qom, I. R. Iran \\ Mohammad Hassan Naderi \\ Faculty of Science \\ Department of Mathematics \\ University of Qom \\ Qom, I. R. Iran
}


Ali Reza Ashrafi

Faculty of Mathematical Siences

Department of Pure Mathematics University of Kashan

Kashan 87317-53153, I. R. Iran

ashrafi@kashanu.ac.ir 